\title{
Solvable scalar and spin models with near-neighbors interactions
}

\author{
A. Enciso, F. Finkel, A. González-López, M.A. Rodríguez \\ Departamento de Física Teórica II, Universidad Complutense, 28040 Madrid, Spain \\ Received 12 September 2004; accepted 10 November 2004 \\ Available online 24 November 2004 \\ Editor: L. Alvarez-Gaumé
}

\begin{abstract}
We construct new solvable rational and trigonometric spin models with near-neighbors interactions by an extension of the Dunkl operator formalism. In the trigonometric case we obtain a finite number of energy levels in the center of mass frame, while the rational models are shown to possess an equally spaced infinite algebraic spectrum. For the trigonometric and one of the rational models, the corresponding eigenfunctions are explicitly computed. We also study the scalar reductions of the models, some of which had already appeared in the literature, and compute their algebraic eigenfunctions in closed form. In the rational cases, for which only partial results were available, we give concise expressions of the eigenfunctions in terms of generalized Laguerre and Jacobi polynomials.
\end{abstract}

(C) 2004 Elsevier B.V. All rights reserved.

PACS: 03.65.Fd; 75.10.Jm

Keywords: Calogero-Sutherland models; Exact solvability; Dunkl operators; Spin chains

Calogero-Sutherland (CS) models are one of the most extensively studied types of exactly solvable and integrable quantum Hamiltonians describing a system of $N$ particles in one dimension with long-range two-body interactions [1-5]. Apart from their intrinsic mathematical relevance [6-8], these models arise naturally in many different fields, such as Yang-Mills theories [9,10], quantum Hall liquids [11], random matrix theory [12-15], propagation of solitons [16], fractional statistics and anyons [17-21], etc. Several generalizations of CS models to particles with internal degrees of freedom ("spin") have been developed over the last decade using two main approaches, namely the supersymmetric formalism [22-24] and the Dunkl operator method [25-30]. Spin CS models are intimately connected with integrable spin chains with long-range position-dependent interactions, like the celebrated Haldane-Shastry spin chain [31,32]. Indeed, when the coupling constant of a spin CS model tends to infinity the particles "freeze" in the classical equilibrium positions of the scalar part of the potential, thus giving rise to a spin chain of Haldane-Shastry (HS) type. This mechanism, usually called the "freezing trick", was first used

E-mail address: artemio@ fis.ucm.es (A. González-López). 
by Polychronakos [33] to construct the first integrals of the original HS spin chain by taking the large coupling constant limit of the corresponding integrals of the Sutherland spin model. These ideas have also been successfully applied to construct integrable and exactly solvable spin chains of HS type from other spin CS models [34-37].

In a recent paper [38], Jain and Khare proposed new solvable versions of the original Calogero and Sutherland scalar models featuring near-neighbors interactions. These new models are closely related to the so-called short-range Dyson models [39,40] in random matrix theory, in that the square of the ground-state wavefunction of the many-body system coincides with the joint probability distribution function for eigenvalues of the corresponding short-range Dyson model. Several generalizations of the Jain and Khare models appeared in a subsequent paper [41], including their extension to the $B C_{N}$ root system and to higher dimensions. The previous publications open a number of interesting questions, such as the existence of other solvable scalar models with near-neighbors interactions, or the construction of spin models with nearneighbors interactions and their corresponding spin chains of Haldane-Shastry type. These short-range spin chains with position-dependent interactions are of particular significance, since they occupy an intermediate position between the well-known Heisenberg chain (short-range, position-independent interactions) and the usual HS-type spin chains (long-range, position-dependent interactions). A first step in this direction is the recent work by Deguchi and Ghosh [42], in which several spin 1/2 models related to the scalar models of Jain and Khare were introduced and partially solved using the supersymmetric formalism. These authors also pointed out how to obtain the spin chains corresponding to these models by applying Polychronakos's freezing trick.

In this Letter we present three new families of solvable scalar and spin $N$-body models with near-neighbors interactions. By contrast with Ref. [42], our approach is based on a modification of the Dunkl operator formalism and provides a wide range of totally explicit solutions for all values of the spin. The potentials of these spin models are given by

$$
\begin{aligned}
& V_{1}=2 a^{2} \sum_{i} \cot \left(x_{i}-x_{i-1}\right) \cot \left(x_{i}-x_{i+1}\right)+2 a \sum_{i} \sin ^{-2}\left(x_{i}-x_{i+1}\right)\left(a-S_{i, i+1}\right), \\
& V_{2}=\omega^{2} r^{2}+\sum_{i} \frac{b(b-1)}{x_{i}^{2}}+\sum_{i} \frac{8 a^{2} x_{i}^{2}}{\left(x_{i}^{2}-x_{i-1}^{2}\right)\left(x_{i}^{2}-x_{i+1}^{2}\right)}+4 a \sum_{i} \frac{x_{i}^{2}+x_{i+1}^{2}}{\left(x_{i}^{2}-x_{i+1}^{2}\right)^{2}}\left(a-S_{i, i+1}\right), \\
& V_{3}=\omega^{2} r^{2}+\sum_{i} \frac{2 a^{2}}{\left(x_{i}-x_{i-1}\right)\left(x_{i}-x_{i+1}\right)}+\sum_{i} \frac{2 a}{\left(x_{i}-x_{i+1}\right)^{2}}\left(a-S_{i, i+1}\right),
\end{aligned}
$$

where $r^{2}=\sum_{i} x_{i}^{2}$ and $a, b>1 / 2$. Here and in what follows the sums run from 1 to $N$, and we are identifying $x_{N+1}$ with $x_{1}$. The operators $S_{i j}$ permute the spin coordinates of the particles $i$ and $j$. In other words, if $\left|s_{1}, \ldots, s_{N}\right\rangle$ (with $-M \leqslant s_{i} \leqslant M, M$ being a half-integer) is an element of the basis of the spin space $\mathcal{S}$, then

$$
S_{i j}\left|\ldots, s_{i}, \ldots, s_{j}, \ldots\right\rangle=\left|\ldots, s_{j}, \ldots, s_{i}, \ldots\right\rangle .
$$

Note that $S_{i j}$ can be expressed in terms of the fundamental SU(2M+1) generators $S_{i}^{a}, a=1, \ldots, 4 M(M+1)$, as $S_{i j}=1 /(2 M+1)+\sum_{a} S_{i}^{a} S_{j}^{a}$.

The spin potentials (1) reduce to solvable scalar potentials by setting $S_{i, i+1}$ to 1 . In particular, the scalar reductions of the potentials (1a) and (1c) are the models introduced by Jain and Khare. The potentials (1a) and (1c) with $M=1 / 2$ are similar to the spin $1 / 2$ potentials introduced in Ref. [42], but differ from them by a spin-dependent term. The spin model (1b), as well as its scalar reduction, are both completely new. There is also a hyperbolic version of the trigonometric potential (1a), obtained by replacing $x_{k}$ by i $x_{k}$ and $V_{1}$ by $-V_{1}$.

The starting point in the solution of the models (1) is the introduction of the following second-order differentialdifference operators, which play the same role as the quadratic combinations of Dunkl operators in the construction 
of CS models with spin [29,30]:

$$
\begin{aligned}
& T_{1}=\sum_{i} z_{i}^{2} \partial_{i}^{2}+2 a \sum_{i} \frac{1}{z_{i}-z_{i+1}}\left(z_{i}^{2} \partial_{i}-z_{i+1}^{2} \partial_{i+1}\right)-2 a \sum_{i} \frac{z_{i} z_{i+1}}{\left(z_{i}-z_{i+1}\right)^{2}}\left(1-K_{i, i+1}\right), \\
& T_{2}=\sum_{i} z_{i} \partial_{i}^{2}+2 a \sum_{i} \frac{1}{z_{i}-z_{i+1}}\left(z_{i} \partial_{i}-z_{i+1} \partial_{i+1}\right)-a \sum_{i} \frac{z_{i}+z_{i+1}}{\left(z_{i}-z_{i+1}\right)^{2}}\left(1-K_{i, i+1}\right), \\
& T_{3}=\sum_{i} \partial_{i}^{2}+2 a \sum_{i} \frac{1}{z_{i}-z_{i+1}}\left(\partial_{i}-\partial_{i+1}\right)-2 a \sum_{i} \frac{1}{\left(z_{i}-z_{i+1}\right)^{2}}\left(1-K_{i, i+1}\right) .
\end{aligned}
$$

Here $\partial_{i} \equiv \partial_{z_{i}}$, the operator $K_{i j}$ permutes the variables $z_{i}$ and $z_{j}$, and we are again identifying $z_{N+1}$ with $z_{1}$. We shall also need in what follows the first-order operators

$$
J_{-}=\sum_{i} \partial_{i}, \quad J_{0}=\sum_{i} z_{i} \partial_{i} .
$$

If $\Phi=\sum_{\mathbf{s}} f_{\mathbf{s}}(\mathbf{z})|\mathbf{s}\rangle$, where $\mathbf{z}=\left(z_{1}, \ldots, z_{N}\right)$ and $\mathbf{s}=\left(s_{1}, \ldots, s_{N}\right)$, is a state totally symmetric under permutations of both the spatial and spin coordinates of each particle, then $K_{i j} \Phi=S_{i j} \Phi$. Hence $T_{\epsilon} \Phi=T_{\epsilon}^{*} \Phi, \epsilon=1,2$, 3, where $T_{\epsilon}^{*}=\left.T_{\epsilon}\right|_{K_{i, i+1} \rightarrow S_{i, i+1}}$. The Hamiltonian $H_{\epsilon}=-\sum_{i} \partial_{x_{i}}^{2}+V_{\epsilon}$ of each of the models (1) can be obtained by applying a suitable gauge transformation and change of variables to a linear combination

$$
\tilde{H}_{\epsilon}=c T_{\epsilon}^{*}+c_{-} J_{-}+c_{0} J_{0}+E_{0} .
$$

More precisely, we can write

$$
H_{\epsilon}=\left.\mu \cdot \tilde{H}_{\epsilon}\right|_{z_{i}=\zeta\left(x_{i}\right)} \cdot \mu^{-1},
$$

where the constants $c, c_{-}, c_{0}, E_{0}$, the gauge factor $\mu$, and the function $\zeta$ are given in each case by

$$
\begin{array}{ll}
\text { (I) } \quad c=4, \quad c_{-}=0, \quad c_{0}=4(1-2 a), \quad E_{0}=2 N a^{2}, \quad \mu=\prod_{i} \sin ^{a}\left(x_{i}-x_{i+1}\right), \quad \zeta(x)=\mathrm{e}^{ \pm 2 \mathrm{i} x}, \\
\text { (II) } \quad c=-4, \quad c_{-}=-2(2 b+1), \quad c_{0}=4 \omega, \quad E_{0}=N \omega(4 a+2 b+1), \\
\mu=\mathrm{e}^{-\frac{\omega}{2} r^{2}} \prod_{i}\left(x_{i}^{2}-x_{i+1}^{2}\right)^{a} x_{i}^{b}, \quad \zeta(x)=x^{2}, \\
\text { (III) } \quad c=-1, \quad c_{-}=0, \quad c_{0}=2 \omega, \quad E_{0}=N \omega(2 a+1), \quad \mu=\mathrm{e}^{-\frac{\omega}{2} r^{2}} \prod_{i}\left(x_{i}-x_{i+1}\right)^{a}, \quad \zeta(x)=x .
\end{array}
$$

The key idea in our approach to the solution of the models (1) is to find an increasing sequence of finitedimensional linear spaces $\tilde{\mathcal{H}}_{\epsilon}^{0} \subset \tilde{\mathcal{H}}_{\epsilon}^{1} \subset \cdots$ invariant under the gauge Hamiltonian $\tilde{H}_{\epsilon}$. Indeed, by Eq. (5) this implies that the corresponding Hamiltonian $H_{\epsilon}$ can be diagonalized in each of its invariant subspaces $\mathcal{H}_{\epsilon}^{n}=\left.\mu \tilde{\mathcal{H}}_{\epsilon}^{n}\right|_{z_{i}=\zeta\left(x_{i}\right)}$, $n=0,1, \ldots$ The operators (2) and (3) preserve the space $\mathcal{P}^{n}$ of polynomials in $\mathbf{z}$ of total degree at most $n$, for all non-negative integer values of $n$. In our recent work on spin CS models $[29,30]$, the operators $T_{\mathrm{CS}}$ analogous to $T_{\epsilon}$ also leave $\mathcal{P}^{n}$ invariant, and in addition commute with the total symmetrizer under particle permutations $\Lambda$. This guarantees that the corresponding operators $T_{\mathrm{CS}}^{*}$ preserve the space of completely symmetric spin functions $\Lambda\left(\mathcal{P}^{n} \otimes \mathcal{S}\right)$. In the present case, however, the operators (2) do not commute with $\Lambda$, and hence it is not clear a priori whether the operators $T_{\epsilon}^{*}$ leave invariant any finite-dimensional space of spin functions. In fact, since $J_{-}$and $J_{0}$ obviously commute with $\Lambda$, the results of Jain and Khare [38] for the scalar case indicate that the operators $T_{1}^{*}$ and $T_{3}^{*}$ possess at least "trivial" invariant spaces of the form $\mathcal{Q} \otimes(\Lambda \mathcal{S})$, where $\mathcal{Q}$ is a finite-dimensional subspace of the space of totally symmetric polynomials in $\mathbf{z}$. As it turns out, each of the operators $T_{\epsilon}^{*}$ preserves a nontrivial 
subspace $\mathcal{T}_{\epsilon}^{n} \subset \Lambda\left(\mathcal{P}^{n} \otimes \mathcal{S}\right)$ for all $n$, namely

$$
\begin{aligned}
& \mathcal{T}_{1}^{n}=\left\langle f\left(\tau_{1}, \tau_{N-1}, \tau_{N}\right) \Lambda \mid s\right\rangle, g\left(\tau_{N-1}, \tau_{N}\right) \Lambda\left(z_{1}|s\rangle\right), q\left(\tau_{1}, \tau_{N}\right) \Lambda\left(z_{1} \cdots z_{N-1}|s\rangle\right) \\
& \quad\left|f_{11}=f_{N-1, N-1}=g_{N-1, N-1}=q_{11}=0\right\rangle, \\
& \mathcal{T}_{2}^{n}=\left\langle f\left(\tau_{1}, \tau_{2}, \tau_{N}\right) \Lambda \mid s\right\rangle, g\left(\tau_{1}, \tau_{N}\right) \Lambda\left(z_{1}|s\rangle\right)\left|f_{22}=f_{N N}=g_{N N}=0\right\rangle, \\
& \mathcal{T}_{3}^{n}=\left\langle f\left(\tau_{1}, \tau_{2}, \tau_{3}\right) \Lambda \mid s\right\rangle, g\left(\tau_{1}, \tau_{2}, \tau_{3}\right) \Lambda\left(z_{1}|s\rangle\right), h\left(\tau_{1}, \tau_{2}\right) \Lambda\left(z_{1}^{2}|s\rangle\right), h\left(\tau_{1}, \tau_{2}\right) \Lambda\left(z_{1} z_{2}\left|s^{\prime}\right\rangle\right)\left|f_{33}=g_{33}=0\right\rangle .
\end{aligned}
$$

An outline of the proof of this statement, which is crucial for what follows, is presented in Appendix A. In Eq. (7),

$$
\tau_{k}=\sum_{i_{1}<\cdots<i_{k}} z_{i_{1}} \cdots z_{i_{k}}
$$

is the $k$ th elementary symmetric polynomial, $f, g, h$, and $q$ are polynomials of total degree in $\mathbf{z}$ less than or equal to $n, n-1, n-2$, and $n-N+1$, respectively, and (for instance) $f_{k}=\partial f / \partial \tau_{k}$. The spin states $|s\rangle \in \mathcal{S}$ are arbitrary, while $\left|s^{\prime}\right\rangle$ denotes a state such that the sum $\sum_{i}\left|s_{i, i+1}^{\prime}\right\rangle$ is totally symmetric, where $\left|s_{i j}^{\prime}\right\rangle$ is defined by $\Lambda\left(z_{1} z_{2}\left|s^{\prime}\right\rangle\right)=\sum_{i<j} z_{i} z_{j}\left|s_{i j}^{\prime}\right\rangle$. Note that the spaces (7) are also preserved by the operator $J_{0}$. Similarly, the operator $J_{-}$leaves the space $\mathcal{T}_{3}^{n}$ invariant, while it preserves $\mathcal{T}_{1}^{n}$ and $\mathcal{T}_{2}^{n}$ provided that $f_{N-1}=f_{N}=g_{N-1}=g_{N}=q=0$ and $f_{N}=g_{N}=0$, respectively. From Eqs. (4) and (6), it easily follows that the gauge Hamiltonians $\tilde{H}_{\epsilon}$ preserve the spaces $\tilde{\mathcal{H}}_{\epsilon}^{n}$ given by

$$
\tilde{\mathcal{H}}_{1}^{n}=\mathcal{T}_{1}^{n}, \quad \tilde{\mathcal{H}}_{2}^{n}=\left.\mathcal{T}_{2}^{n}\right|_{f_{N}=g_{N}=0}, \quad \tilde{\mathcal{H}}_{3}^{n}=\mathcal{T}_{3}^{n} .
$$

By diagonalizing the Hamiltonian $H_{\epsilon}$ in its corresponding invariant spaces $\mathcal{H}_{\epsilon}^{n}, n=0,1, \ldots$, one can in principle construct an infinite sequence of exact eigenvalues and eigenfunctions, which shall be referred to as "algebraic" in what follows. We have found in this way all the algebraic eigenvalues of the spin models (1). We also present explicit expressions for the corresponding algebraic eigenfunctions, with the only exception of the spin eigenfunctions of the model (1c) not factorizing as $\mu f\left(\tau_{1}, \tau_{2}, \tau_{3}\right) \Lambda|s\rangle$. In particular, we obtain all the algebraic eigenfunctions of the scalar reductions of the spin potentials (1), thus considerably extending the results of Refs. [38,41]. It should be noted, however, that the point spectrum could possibly include additional eigenvalues and eigenfunctions which are not algebraic. We shall now discuss in more detail each of the models (1).

Case 1. The Hamiltonian $H_{1}$ commutes with the total linear momentum $P=-\mathrm{i} \sum_{k} \partial_{x_{k}}$, so that it admits a basis of eigenfunctions with well-defined total momentum. In fact, since in this case $\tau_{N}^{l}=\exp \left( \pm 2 \mathrm{i} l \sum_{k} x_{k}\right)$, multiplying an eigenfunction of $H_{1}$ with energy $E$ and total momentum $p$ by $\tau_{N}^{l}$ simply "boosts" its energy and total momentum. We shall take advantage of this fact to "normalize" the algebraic eigenfunctions to zero total momentum. It easily follows from Eq. (7a) that when this normalization is performed one obtains only a finite number of eigenfunctions of $H_{1}$. In the scalar case, there are exactly four eigenfunctions with zero momentum, namely

$$
\psi_{0}=\mu, \quad \psi_{1,2}=\mu \sum_{i}\left\{\begin{array}{c}
\cos \\
\sin
\end{array}\right\}\left(2\left(x_{i}-X\right)\right), \quad \psi_{3}=\mu\left[\frac{N a}{2 a+1}+\sum_{i<j} \cos \left(x_{i}-x_{j}\right)\right],
$$

where $X=\frac{1}{N} \sum_{i} x_{i}$ is the center of mass coordinate. Their respective energies are $E_{0}$ (ground state), $E_{1,2}=$ $E_{0}+4(2 a-1+1 / N)$, and $E_{3}=E_{0}+8(2 a+1)$. These are essentially the solutions found in [43].

In the spin case, to each scalar eigenfunction (10) there correspond $\left(\begin{array}{c}2 M+N \\ N\end{array}\right)$ factorized solutions of the form $\Psi_{n}^{(0)}=\psi_{n} \Lambda|s\rangle$, where $|s\rangle \in \mathcal{S}$ is arbitrary. There are three additional families of algebraic spin eigenfunctions with zero total momentum, given by

$$
\Psi_{1,2}^{(1)}=\mu \sum_{i}\left\{\begin{array}{c}
\cos \\
\sin
\end{array}\right\}\left(2\left(x_{i}-X\right)\right)\left|s_{i}\right\rangle, \quad \Psi_{3}^{(1)}=\mu\left[\frac{a}{2 a+1} \sum_{i}\left|s_{i}\right\rangle+\sum_{i<j} \cos \left(x_{i}-x_{j}\right)\left|s_{j}\right\rangle\right],
$$


where the spin states $\left|s_{i}\right\rangle$ are defined by $\Lambda\left(z_{1}|s\rangle\right)=\sum_{i} z_{i}\left|s_{i}\right\rangle$, and $|s\rangle \in \mathcal{S}$ is any non-symmetric state. It should be noted that each of the spin states $\Psi_{n}^{(0)}$ and $\Psi_{n}^{(1)}$ has the same energy as the scalar eigenfunction $\psi_{n}$.

Case 2. In this case the algebraic energies are given by $E_{n}=E_{0}+n c_{0}=E_{0}+4 n \omega$, where the quantum number $n=0,1, \ldots$ is the degree in $\mathbf{z}$ of the corresponding eigenfunction of the gauged Hamiltonian $\tilde{H}_{2}$. This follows easily from the fact that both $T_{2}^{*}$ and $J_{-}$lower the degree, while $J_{0}$ preserves it. As for Calogero's original model, the algebraic spectrum is equally spaced, but the spacing is twice the value suggested by the harmonic term. Unlike the usual CS models, the algebraic levels of this model have a well-defined thermodynamic limit, i.e., $E_{n} / N \rightarrow \omega(4 a+2 b+1)$ as $N \rightarrow \infty$. This property, which was already noted in Ref. [38] for the scalar reductions of the potentials (1a) and (1c), is in fact shared by all the models (1). In the scalar case, for each $n \geqslant 2$ there are two algebraic eigenfunctions with energy $E_{n}$, namely

$$
\begin{aligned}
& \psi_{n}^{(0)}=\mu L_{n}^{\alpha-1}\left(\omega r^{2}\right), \quad n=0,1, \ldots, \\
& \psi_{n}^{(1)}=\mu\left[\omega^{2}\left(N(\alpha+1) \sum_{i} x_{i}^{4}-\alpha r^{4}\right) L_{n-2}^{\alpha+3}\left(\omega r^{2}\right)+n(n-1) \alpha L_{n}^{\alpha-1}\left(\omega r^{2}\right)\right], \quad n=2,3, \ldots,
\end{aligned}
$$

where $\alpha=N(2 a+b+1 / 2)$ and $L_{v}^{\lambda}$ is a generalized Laguerre polynomial of degree $v$. Note, in particular, that $\psi_{0}^{(0)}=\mu$ has no nodes in the configuration space $0<x_{1}<\cdots<x_{N}$, and is thus the ground state wavefunction. In the spin case, for each energy level $E_{n}$ we have first of all the factorized eigenfunctions of the form $\Psi_{n}^{(k)}=$ $\psi_{n}^{(k)} \Lambda|s\rangle, k=0,1$. In addition, for each $n \geqslant 1$ there is a family of genuine spin eigenfunctions

$$
\Psi_{n}^{(2)}=\mu\left[L_{n-1}^{\alpha+1}\left(\omega r^{2}\right) \Lambda\left(x_{1}^{2}|s\rangle\right)-\frac{\alpha}{N \omega} L_{n-1}^{\alpha}\left(\omega r^{2}\right) \Lambda|s\rangle\right]
$$

where $|s\rangle \in \mathcal{S}$ is non-symmetric. Note that, as in the previous case, the spin eigenfunctions with lowest algebraic energy $E_{0}$ are all factorized states.

Case 3. This is probably the most interesting case, since the algebraic eigenfunctions depend essentially on the three symmetric variables $\tau_{1}, \tau_{2}, \tau_{3}$. As in the previous case, the algebraic energies are given by $E_{n}=E_{0}+n c_{0}=$ $E_{0}+2 n \omega$, where $n$ is again the degree in $\mathbf{z}$ of the corresponding eigenfunctions of $\tilde{H}_{3}$. We shall begin, as usual, with the scalar case, for which we have been able to compute all the algebraic eigenfunctions in closed form. For each energy level $E_{n}$ with $n \geqslant 3$ there are two infinite families of algebraic eigenfunctions $\psi_{l m}^{(k)}, k=0,1$, with $n=2 l+m$. The first family is given by

$$
\psi_{l m}^{(0)}=\mu \tau_{1}^{m} L_{l}^{-\beta}\left(\omega r^{2}\right) P_{\left[\frac{m}{2}\right]}^{(\alpha, \beta)}(t), \quad l, m=0,1, \ldots,
$$

where $t=2 N r^{2} / \tau_{1}^{2}-1, \alpha=N(a+1 / 2)-3 / 2, \beta \equiv \beta(m)=1-m-N(a+1 / 2), P_{v}^{(\alpha, \beta)}$ is a Jacobi polynomial of degree $v$, and $[x]$ denotes the integer part of $x$. The second family reads

$$
\psi_{l m}^{(1)}=\mu \tau_{1}^{m-3} L_{l}^{-\beta}\left(\omega r^{2}\right)\left[P_{\left[\frac{m-3}{2}\right]}^{(\alpha+3, \beta)}(t) \sum_{i} x_{i}^{3}+\frac{3 \tau_{1}^{3}}{2 N^{2}} \varphi_{l m}(t)\right], \quad l, m-3=0,1, \ldots,
$$

where $\varphi_{l m}$ is a polynomial of degree $[m / 2]$ given by

$$
\varphi_{l m}=\frac{m+2 \alpha+2}{m-1} P_{\frac{m}{2}}^{(\alpha+2, \beta-2)}(t)-P_{\frac{m}{2}-1}^{(\alpha+3, \beta-1)}(t)-\frac{4 \alpha+7}{m-1} P_{\frac{m}{2}-1}^{(\alpha+2, \beta-1)}(t)+\frac{1}{3} P_{\frac{m}{2}-2}^{(\alpha+3, \beta)}(t),
$$

for even $m$, while for odd $m$ we have

$$
\begin{aligned}
\varphi_{l m}= & 2 P_{\frac{m-1}{2}}^{(\alpha+2, \beta-1)}(t)-P_{\frac{m-1}{2}}^{(\alpha+3, \beta-1)}(t)+\frac{1}{3} P_{\frac{m-3}{2}}^{(\alpha+3, \beta)}(t) \\
& +\frac{m+2 \alpha+2}{m(m-2)} P_{\frac{m-3}{2}}^{(\alpha+1, \beta)}(t)-\frac{m+2 \alpha+2}{m-2} P_{\frac{m-3}{2}}^{(\alpha+2, \beta)}(t) .
\end{aligned}
$$


Note that, as in the previous cases, $\psi_{0}^{(0)}=\mu$ is the ground state. The above results show that for all $N \geqslant 3$ the scalar reduction of the Hamiltonian $H_{3}$ possesses two different families of eigenfunctions of the form $\mu L_{l}^{-\beta}\left(\omega r^{2}\right) p_{\nu}(\mathbf{x})$, with $p_{v}$ a homogeneous polynomial of degree $v \geqslant 3$, cf. Eqs. (11) and (12)-(14). This was verified only up to $v=6$ and $N \geqslant v$ in Ref. [41].

In the spin case, the algebraic energies are of course the same as in the scalar case. As in the previous cases, each scalar algebraic eigenfunction gives rise to $\left(\begin{array}{c}2 M+N \\ N\end{array}\right)$ factorized spin eigenfunctions. The computation of the remaining algebraic spin eigenfunctions, which is considerably more involved than in Cases 1 and 2, is still in progress. Even without an explicit knowledge of the eigenfunctions, for each algebraic level $E_{n}$ one can easily compute the number of independent states of the form (7c) of degree $n$. If all the eigenfunctions of this model (and of its scalar reduction) were algebraic, the previous remark would imply that the degeneracy of the levels can be explicitly found. The method of Ref. [37] could then be used to compute the partition function of the associated spin chain

$$
\mathrm{H}_{3}=\sum_{i}\left(\xi_{i}-\xi_{i+1}\right)^{-2} S_{i, i+1}
$$

where $\left(\xi_{1}, \ldots, \xi_{N}\right)$ is an equilibrium of the scalar potential

$$
U_{3}=\frac{1}{2} r^{2}+\sum_{i} \frac{1}{\left(x_{i}-x_{i-1}\right)\left(x_{i}-x_{i+1}\right)}+\sum_{i} \frac{1}{\left(x_{i}-x_{i+1}\right)^{2}} .
$$

This remark obviously applies to the rational model (1b) as well. Note, however, that for this model one can in addition construct states of the associated spin chain by applying the freezing trick to the genuine spin eigenfunctions $\Psi_{n}^{(2)}$ presented above.

\section{Acknowledgements}

This work was partially supported by the Spanish DGI under grant No. BFM2002-02646. A.E. acknowledges the financial support of the Spanish Ministry of Education through an FPU scholarship.

\section{Appendix A. Invariance of the spaces $\mathcal{T}_{\epsilon}^{n}$ under the operators $T_{\epsilon}^{*}$}

The proof of the invariance of the spaces $\mathcal{T}_{\epsilon}^{n}$ in Eq. (7) under the corresponding operators $T_{\epsilon}^{*}$ is a lengthy but essentially straightforward calculation. For the reader's convenience, we shall outline in this appendix the proof for the space $\mathcal{T}_{1}^{n}$, the other cases being fairly similar.

Note, first of all, that $T_{1} \Phi=T_{1}^{*} \Phi$ for all $\Phi \in \mathcal{T}_{1}^{n}$, since all the states in this space are completely symmetric under permutations. Let us begin by considering the action of $T_{1}$ on factorized states of the form $f \Lambda|s\rangle$, where $f$ belongs to the finite-dimensional space of symmetric polynomials

$$
\mathcal{Q}_{1}^{n}=\left\langle f\left(\tau_{1}, \tau_{N-1}, \tau_{N}\right) \mid f_{11}=f_{N-1, N-1}=0, \operatorname{deg} f \leqslant n\right\rangle,
$$

the symmetric variables $\tau_{k}$ are defined in Eq. (8), $f_{j k \ldots l}=\partial_{\tau_{j}} \partial_{\tau_{k}} \cdots \partial_{\tau_{l}} f$, and deg $f$ is the degree of $f$ in $\mathbf{z}$. Since $T_{1}(f \Lambda|s\rangle)=\left(T_{1} f\right) \Lambda|s\rangle$, we must show that the scalar space $\mathcal{Q}_{1}^{n}$ is invariant under $T_{1}$. If $f \in \mathcal{Q}_{1}^{n}$, then $K_{i j} f=f$ and thus

$$
T_{1} f=\left(\sum_{i} z_{i}^{2} \partial_{i}^{2}+2 a X_{1}\right) f
$$


where

$$
X_{1}=\sum_{i} \frac{1}{z_{i}-z_{i+1}}\left(z_{i}^{2} \partial_{i}-z_{i+1}^{2} \partial_{i+1}\right) .
$$

From the identities

$$
\begin{aligned}
& X_{1} \tau_{1}=\sum_{i} \frac{z_{i}^{2}-z_{i+1}^{2}}{z_{i}-z_{i+1}}=2 \tau_{1}, \\
& X_{1} \tau_{N}=\tau_{N} \sum_{i}\left(\frac{z_{i}}{z_{i}-z_{i+1}}-\frac{z_{i+1}}{z_{i}-z_{i+1}}\right)=N \tau_{N}, \\
& X_{1} \tau_{N-1}=\sum_{i} X_{1}\left(\frac{\tau_{N}}{z_{i}}\right)=N \sum_{i} \frac{\tau_{N}}{z_{i}}-\tau_{N} \sum_{i} \frac{X_{1} z_{i}}{z_{i}^{2}}=N \tau_{N-1}+\sum_{i}\left(\frac{1}{z_{i}-z_{i+1}}-\frac{1}{z_{i-1}-z_{i}}\right)=N \tau_{N-1},
\end{aligned}
$$

it follows that

$$
X_{1} f=2 \tau_{1} f_{1}+N \tau_{N-1} f_{N-1}+N \tau_{N} f_{N} .
$$

On the other hand, the elementary identity

$$
\partial_{i} f\left(\tau_{1}, \tau_{N-1}, \tau_{N}\right)=f_{1}+\left(z_{i}{ }^{-1} \tau_{N-1}-z_{i}^{-2} \tau_{N}\right) f_{N-1}+z_{i}{ }^{-1} \tau_{N} f_{N}
$$

implies that

$$
\begin{array}{rl}
\sum_{i} z_{i}^{2} \partial_{i}^{2} & f\left(\tau_{1}, \tau_{N-1}, \tau_{N}\right) \\
= & \left(\tau_{1}^{2}-2 \tau_{2}\right) f_{11}+2\left(\tau_{1} \tau_{N-1}-N \tau_{N}\right) f_{1, N-1}+2 \tau_{1} \tau_{N} f_{1 N} \\
& \quad+\left[(N-1) \tau_{N-1}^{2}-2 \tau_{N-2} \tau_{N}\right] f_{N-1, N-1}+2(N-1) \tau_{N-1} \tau_{N} f_{N-1, N}+N \tau_{N}^{2} f_{N N} .
\end{array}
$$

By Eqs. (A.3) and (A.5), the conditions $f_{11}=f_{N-1, N-1}=0$ guarantee that $T_{1} f$ belongs to $\mathcal{Q}_{1}^{n}$. Therefore, the subspace $\mathcal{Q}_{1}^{n} \otimes(\Lambda \mathcal{S})$ of factorized states in $\mathcal{T}_{1}^{n}$ is invariant under the action of $T_{1}$.

Let us turn next to the states of the form $g\left(\tau_{N-1}, \tau_{N}\right) \Lambda\left(z_{1}|s\rangle\right) \equiv g \Phi$, where $g$ is a polynomial of degree in $\mathbf{z}$ at most $n-1$ satisfying $g_{N-1, N-1}=0$. Writing $\Phi=\sum_{i} z_{i}\left|s_{i}\right\rangle$, we have

$$
T_{1}(g \Phi)=\sum_{i} T_{1}\left(g z_{i}\right)\left|s_{i}\right\rangle .
$$

On the other hand,

$$
T_{1}\left(g z_{i}\right)=\left(T_{1} g\right) z_{i}+2 z_{i}^{2} \partial_{i} g+2 a g\left(\frac{z_{i}^{2}}{z_{i}-z_{i+1}}+\frac{z_{i}^{2}}{z_{i}-z_{i-1}}-\frac{z_{i} z_{i+1}}{z_{i}-z_{i+1}}-\frac{z_{i} z_{i-1}}{z_{i}-z_{i-1}}\right),
$$

and therefore, by Eqs. (A.3)-(A.5),

$$
\begin{aligned}
T_{1}(g \Phi)= & {\left[2(N-1) \tau_{N-1} \tau_{N} g_{N-1, N}+N \tau_{N}^{2} g_{N N}\right.} \\
& \left.+2(a N+1)\left(\tau_{N-1} g_{N-1}+\tau_{N} g_{N}\right)+4 a g\right] \Phi-2 \tau_{N} g_{N-1} \Lambda|s\rangle .
\end{aligned}
$$

Hence $T_{1}(g \Phi)$ belongs to $\mathcal{T}_{1}^{n}$, as claimed.

Consider, finally, states of the form $q\left(\tau_{1}, \tau_{N}\right) \Lambda\left(z_{1} \cdots z_{N-1}|s\rangle\right) \equiv q \Phi$, where $q$ is a polynomial of degree at most $n-N+1$ in $\mathbf{z}$ satisfying $q_{11}=0$. Since

$$
\Phi=\tau_{N} \Lambda\left(z_{1}^{-1}|s\rangle\right) \equiv \tau_{N} \sum_{i} z_{i}^{-1}\left|s_{i}\right\rangle,
$$


calling $\hat{q}=\tau_{N} q$ we have

$$
T_{1}(q \Phi)=\sum_{i} T_{1}\left(\frac{\hat{q}}{z_{i}}\right)\left|s_{i}\right\rangle=\sum_{i}\left[\left(T_{1} \hat{q}\right) z_{i}^{-1}-2 \partial_{i} \hat{q}+2 \hat{q} z_{i}^{-1}\right]\left|s_{i}\right\rangle=\tau_{N}^{-1}\left(T_{1} \hat{q}\right) \Phi-2 \sum_{i} \partial_{i} \hat{q}\left|s_{i}\right\rangle+2 q \Phi .
$$

Using Eqs. (A.3) and (A.5) we easily obtain

$$
\tau_{N}^{-1} T_{1} \hat{q}=2 \tau_{1} \hat{q}_{1 N}+N \tau_{N} \hat{q}_{N N}+2 a\left(2 \tau_{1} q_{1}+N \hat{q}_{N}\right),
$$

and therefore (cf. Eq. (A.4))

$$
T_{1}(q \Phi)=\left[2 \tau_{1} \hat{q}_{1 N}+N \tau_{N} \hat{q}_{N N}+2(a N-1) \hat{q}_{N}+4 a \tau_{1} q_{1}+2 q\right] \Phi-2 \hat{q}_{1} \Lambda|s\rangle
$$

clearly belongs to $\mathcal{T}_{1}^{n}$.

\section{References}

[1] F. Calogero, J. Math. Phys. 12 (1971) 419.

[2] B. Sutherland, Phys. Rev. A 4 (1971) 2019.

[3] B. Sutherland, Phys. Rev. A 5 (1972) 1372.

[4] M.A. Olshanetsky, A.M. Perelomov, Phys. Rep. 94 (1983) 313.

[5] J.F. van Diejen, L. Vinet (Eds.), Calogero-Moser-Sutherland Models, CRM Series in Mathematical Physics, Springer-Verlag, New York, 2000.

[6] A. Kasman, Commun. Math. Phys. 172 (1995) 427.

[7] L. Lapointe, L. Vinet, Commun. Math. Phys. 178 (1996) 425.

[8] T.H. Baker, P.J. Forrester, Commun. Math. Phys. 188 (1997) 175.

[9] A. Gorsky, N. Nekrasov, Nucl. Phys. B 414 (1994) 213.

[10] E. D’Hoker, D.H. Phong, Nucl. Phys. B 513 (1998) 405.

[11] H. Azuma, S. Iso, Phys. Lett. B 331 (1994) 107.

[12] B. Sutherland, J. Math. Phys. 12 (1971) 246.

[13] B. Sutherland, J. Math. Phys. 12 (1971) 251.

[14] B.D. Simons, P.A. Lee, B.L. Altshuler, Phys. Rev. Lett. 72 (1994) 64.

[15] N. Taniguchi, B.S. Shastry, B.L. Altshuler, Phys. Rev. Lett. 75 (1995) 3724.

[16] A.P. Polychronakos, Phys. Rev. Lett. 74 (1995) 5153.

[17] A.P. Polychronakos, Nucl. Phys. B 324 (1989) 597.

[18] Z.N.C. Ha, Phys. Rev. Lett. 73 (1994) 1574.

[19] Z.N.C. Ha, Nucl. Phys. B 435 (1995) 604.

[20] A.L. Carey, E. Langmann, Commun. Math. Phys. 201 (1999) 1.

[21] P. Shukla, Phys. Rev. Lett. 87 (2001) 194102(4).

[22] L. Brink, A. Turbiner, N. Wyllard, J. Math. Phys. 39 (1998) 1285.

[23] P. Desrosiers, L. Lapointe, P. Mathieu, Nucl. Phys. B 606 (2001) 547.

[24] P.K. Ghosh, Nucl. Phys. B 595 (2001) 519.

[25] C.F. Dunkl, Trans. Am. Math. Soc. 311 (1989) 167.

[26] A.P. Polychronakos, Phys. Rev. Lett. 69 (1992) 703.

[27] T. Yamamoto, Phys. Lett. A 208 (1995) 293.

[28] B. Basu-Mallick, Nucl. Phys. B 482 (1996) 713.

[29] F. Finkel, D. Gómez-Ullate, A. González-López, M.A. Rodríguez, R. Zhdanov, Commun. Math. Phys. 221 (2001) 477.

[30] F. Finkel, D. Gómez-Ullate, A. González-López, M.A. Rodríguez, R. Zhdanov, Nucl. Phys. B 613 (2001) 472.

[31] F.D.M. Haldane, Phys. Rev. Lett. 60 (1988) 635.

[32] B.S. Shastry, Phys. Rev. Lett. 60 (1988) 639.

[33] A.P. Polychronakos, Phys. Rev. Lett. 70 (1993) 2329.

[34] A.P. Polychronakos, Nucl. Phys. B 419 (1994) 553.

[35] D. Bernard, V. Pasquier, D. Serban, Europhys. Lett. 30 (1995) 301.

[36] T. Yamamoto, O. Tsuchiya, J. Phys. A 29 (1996) 3977.

[37] A. Enciso, F. Finkel, A. González-López, M.A. Rodríguez, hep-th/0406054, Nucl. Phys. B, in press.

[38] S.R. Jain, A. Khare, Phys. Lett. A 262 (1999) 35. 
[39] B. Grémaud, S.R. Jain, J. Phys. A 31 (1998) L637.

[40] E.B. Bogomolny, U. Gerland, C. Schmit, Phys. Rev. E 59 (1999) R1315.

[41] G. Auberson, S.R. Jain, A. Khare, J. Phys. A 34 (2001) 695.

[42] T. Deguchi, P.K. Ghosh, J. Phys. Soc. Jpn. 70 (2001) 3225.

[43] M. Ezung, N. Gurappa, A. Khare, P.K. Panigrahi, cond-mat/0007005. 